\title{
Blogging to Disseminate Research
}

\author{
Sharing Results with Communities and Partners
}

\author{
Christina Nelson \\ University of Washington Tacoma \\ Tacoma, WA, USA \\ cnn1996@uw.edu
}

\author{
Emma J. Rose \\ University of Washington Tacoma \\ Tacoma, WA, USA \\ ejrose@uw.edu
}

\begin{abstract}
While blogging has become a popular social tool, less work has focused on how blogging can be used as a tool for researchers to disseminate research findings to the public. In this experience report, we share how we created a research blog to disseminate information about a multi-year research project to develop a social robot that will be used in educational settings. The report begins with our goals in creating a research blog, including the desire to keep community partners and the broader audience for our research project engaged and informed. We describe our approach to developing and maintaining a research blog and related social media accounts and discuss its impact. We reflect on the process of using a blog to share research findings with the public. We conclude by providing a list of best practices for other teams considering using a blog to share emerging results.
\end{abstract}

\section{CCS CONCEPTS}

- Information systems $\rightarrow$ Multimedia content creation; • Humancentered computing $\rightarrow$ User centered design.

\section{KEYWORDS}

blogging, research dissemination, social media

\section{ACM Reference Format:}

Christina Nelson and Emma J. Rose. 2019. Blogging to Disseminate

Research: Sharing Results with Communities and Partners. In Proceedings of SIGDOC '19: ACM Conference on the Design of Communication (SIGDOC 19). ACM, New York, NY, USA, 6 pages.

https://doi.org/10.1145/3328020.3353945

\section{INTRODUCTION}

Large academic research projects are often complex, lengthy, and include multidisciplinary team members that change over time. It can be a challenge to capture emerging knowledge, document activities, and share that information with stakeholders and the public. Oftentimes the results of research are solely shared in academic and scholarly publications. However, when a project involves a large group of participants and community partners, conceiving of ways to disseminate research can help share emerging findings with a larger audience who may be invested or interested in the

Permission to make digital or hard copies of all or part of this work for personal or classroom use is granted without fee provided that copies are not made or distributed for profit or commercial advantage and that copies bear this notice and the full citation on the first page. Copyrights for components of this work owned by others than the author(s) must be honored. Abstracting with credit is permitted. To copy otherwise, or republish, to post on servers or to redistribute to lists, requires prior specific permission and/or a fee. Request permissions from permissions@acm.org.

SIGDOC 19, Oct 04-06, 2019, Portland, OR

(c) 2019 Copyright is held by the owner/author(s). Publication rights licensed to ACM. ACM 978-1-4503-6790-5/19/10 ..\$15.00

https://doi.org/10.1145/3328020.3353945 results. For the past three years, our team has used a research blog to disseminate information and data about an ongoing, large scale, research project.

Blogging is a beneficial way to communicate emerging research results. Because blog posts encourage a less formal tone, researchers can write and publish information that is easy to access and understand by a wide audience. Further, blogging and communicating via social media gives researchers a tool to connect with interested members of the public by rapidly sharing findings that are immediately accessible $[2,5]$. However, maintaining a research blog can be difficult because of time constraints [4] and when not updated consistently, can feel stale. Further, multiple authors can impact issues of consistency and voice. While blogging has gained popularity as a social tool, there has been minimal work discussing how blogs can be used by research teams to disseminate findings at all stages in the process.

In this experience report, we share how we developed a research blog to share emerging research findings to disseminate information about a large scale, multi-year research project involving community partners and a large team of student researchers. The research project involves the design and development of a social robot for the use in educational settings. First, we share the goals of creating a blog including the desire to follow a participatory and ethical relationship with our research partners and communities and also to help document and share our work in process at all stages in the design. Second, we describe the approach to developing, maintaining, and updating a research blog and related social media accounts, including editorial direction, recruitment of authors, and the creation of a style guide. Third, we reflect on the successes and challenges of the blog as a research dissemination tool. We conclude by providing a list of best practices for other teams considering using a blog to share emerging results.

\section{BACKGROUND ON THE RESEARCH PROJECT}

Project EMAR is an interdisciplinary project at University of Washington to develop a social robot to address and intervene in teen stress that will be used in school settings.

Today's adolescents experience more stress than any other age group $[1,3]$. However, while a great deal of research has been conducted on human-robot interaction among children, little research has examined the interaction between robots and teenagers. Project EMAR is working to develop an engaging social robot that will live in a high school and provide anonymous and aggregate data on teen stress. In order to effectively measure teen stress in a public high school environment, our social robot uses Ecological Momentary Assessment (EMA) [8] to capture stress data and offer a micro-intervention. 
Our team utilizes a participatory design approach to engage teens in the development of our social robot [6]. Our team partners with school districts in Tacoma and Seattle in Washington State in the US to co-design robots with teens.

Given that the project is a large scale, multi-year, federallyfunded project, it can be complex to communicate about the emerging developments. The project scales two campuses, three academic departments, multiple faculty and research scientists, and undergraduates and graduate students from a wide variety of disciplines. Further, as a project rooted in participatory design that engages with community partners in the form of schools, teachers, principals, teens, and their parents, we have a commitment to communicate with our partners in ways that reflect openness, transparency, and reciprocity.

\section{GOALS OF THE RESEARCH BLOG}

In order to disseminate emerging research findings to our community partners, our team created the Project EMAR Blog in 2016 in addition to a Twitter and Facebook page (https://sites.uw.edu/emar/).

Blog posts are written for a variety of audiences. The blog has three priority audiences each with unique needs. The first audience is our community partners. This could be teens who have taken part in the research, teachers or administrators at our partner schools, or parents. They might be coming to the blog to see what we learned during our visit to their school or potentially decide if they want to take part in additional activities. In sharing our blog posts on a public platform, our team intends to give our community partners access to information about our project in an accessible way. The second audience includes researchers in related fields who are interested in what the project is about and what we are learning along the way. In a field $l$ ike $h$ uman-robot interaction, where the pace of research moves quickly, it is helpful to provide this ongoing view of our work, methods, and preliminary findings. Further, students who are interested in getting engaged in our research may be looking at the blog to see what is like to work on our team. Finally, we consider the general public as a final primary audience, this represents people outside of our research community and community partnerships that may be interested in learning more about the project.

The purpose of disseminating research findings on an accessible public blog is to create and maintain a participatory and ethical relationship with our community partners. In operating a blog that is accessible to the public, our team aims to provide constant updates on our progress in real-time, ensuring that all stages of the research process are documented for our community partners to follow so that we remain transparent at all points in the process. Further, our blog post platforms also allow our team to leverage media opportunities, connecting our research to internal media sources at the university as well as external media sources that are interested in learning more about our research project. By disseminating our research on social media platforms, we intend for our blog to connect our team with media sources who would be interested in learning more about our project to develop a social robot with teens that will address teen stress.

Our social media sites on Facebook and Twitter allow us to further disseminate and extend the reach of our blog by pushing out updates to across social networks which are then engaged with by others in our institution, communities, and other researchers in the fields of design and social robotics.

\section{APPROACH TO RESEARCH BLOGGING}

Over the course of the past three years, our research team has published 47 blog posts. Each blog post provides the audiences with information on our latest research activities, including the research questions we aimed to answer, the procedures used to collect and analyze data, and the emerging results.

The blog posts we share with the public are visually heavy, containing pictures from our interactions with our community partners. Blog posts are a maximum of 1000 words in length. In this section, we provide details about authorship, audience and tone, developing a blog post style guide, and sharing blog posts on social media.

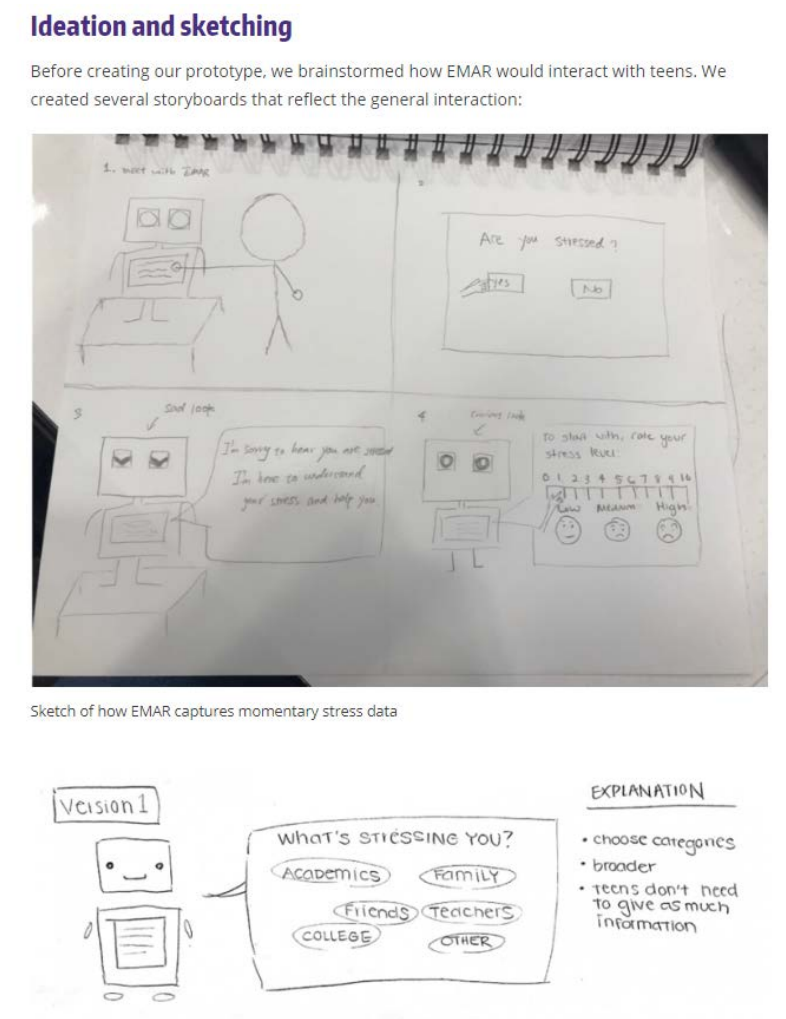

Figure 1: A blog post published on our website uses pictures of student work to share the design process with the public.

\subsection{Authoring, editing, and publishing}

Our research team is a cross-campus, multidisciplinary team of undergraduate and graduate students at the University of Washington Seattle and University of Washington Tacoma campuses. To share the broad range of voices and perspectives on our research team, all researchers participating on the project are asked to author one blog post, per 10 week quarter. Blog posts are written in teams 
of no more than three students. Student teams select a research topic to cover at the beginning of each quarter from a list of blog post topics and sign up for a submission date on a shared blog post schedule. Student authors are asked to reference a blog post style guide throughout the process of authoring a blog in order to ensure that their blog post meets the standards for formatting and style. Once the team of students has finished a first draft of their blog post, they send it to the blog editor, a position held by a student, to receive feedback on their initial draft. Blogging is an iterative process and teams receive feedback and suggestions for edits from the editing team throughout the writing process. After the student editor has finished editing through the blog post draft, the draft is sent to one of the Principal Investigators on the research team who reviews and provides minor edits on blog post for consistency in tone and voice and gives additional feedback on structure, word choice, and clarity. After the post has been revised, the student editor publishes the post on a university blog site and shares the blog post with the public on the research team's Facebook and Twitter pages. Blog post content is produced on a bi-weekly basis throughout the academic year to provide the public with frequent updates on our research progress.

\subsection{Audience and tone}

Disseminating our research on a public blog platforms allows audiences who would not typically be reached by academic papers to gain access to our research findings. Our blog posts are written for members of the public who are interested in learning more about our project to develop a social robot that will address teen stress in a local high school. Blog posts are written for researchers outside of the project who are interested in learning about engaging communities in research using participatory design best practices. Our audience also includes the community partners who we work with in high schools in the Pacific Northwest region of the US, including teenagers, parents and faculty members at our partner high schools.

Because of their casual, yet informative tone, blog posts differ from academic papers and aim to educate members of the public on current research findings in a lighthearted manner. Blog posts contain sentences that are brief and to the point, using simple vocabulary to convey our findings without overwhelming readers with domain-specific jargon. Blog posts are written in a conversational manner, focusing on one specific and meaningful research topic that our team is interested in gaining insight into. Rather than depending on heavy text-based descriptions, our blog posts maintain a casual tone as they integrate 3-5 images per post from our visits to the field to contextualize the research process for our readers. Blog posts are written in active voice and use personal pronouns.

\subsection{Blog post style guide}

Due to the multiple authors contributing to the blog, we developed a blog post style guide to streamline the process and to maintain consistency across multiple posts.

Authors reference a blog post style guide to adhere to the format of the blog. The blog post style guide provides members of the team with guidelines on addressing elements including:

- Writing for a target audience
- Identifying blog post purpose

- Remaining consistent in voice and tone

- Creating effective headlines

- Structuring the document

- Integrating visuals

The style guide provides contextual examples of how each of these elements might look in the context of our blog, referencing previous posts as examples. Sharing the style guide in Google Documents allows our team to make changes as necessary while ensuring that all members of our research team have access to the latest additions. When authors have additional questions about writing style and approaching a blog topic that are not contained in the blog post style guide, they are asked to reach out to one of the editors of the blog. We envision the style guide as a living document that has been created and revised over time. As the team has grown, the style guide helps to maintain consistency and provide efficient ways for new authors to quickly get up to speed.

\subsection{Sharing posts on social media}

Social media platforms serve as a powerful resource for researchers to quickly disseminate research findings to the public [2]. To connect our research findings to our community partners, our team maintains a Twitter and Facebook account that we use to share blog posts with the public.

When sharing posts with the public, our team connects with our community partners by tagging the usernames of our community partners in each of our posts. In addition to tagging usernames to connect our blog posts with interested members of the public, we use domain-specific hashtags related to social robotics, stress, and user-centered design. In using these social functions, our team is able to use our online platforms to provide access to the research findings to members of the public who might be interested in participating on our project in the future.

Disseminating research on Twitter drives the impact of sharing research findings, engaging groups who lack access to academic journals with access to research findings [7]. In sharing our research findings on social media, we are able to remain transparent with the community partners that we research with, including teenagers, parents, and faculty members in the Pacific Northwest.

\section{EVALUATING THE IMPACT OF THE BLOG}

In this section, we report on the impact of the blog in relation to our external audience audience engagement metrics and also on the impact on our project team. Our team monitored the traffic on our blog website in addition to Twitter analytics to understand audience experiences with the content we developed.

\subsection{Success metrics}

To evaluate the effectiveness of our research blog, our team used Google Analytics to track web traffic overtime. In addition, we monitor likes and retweets accumulated on our Twitter account. In sharing research on social media, we also looked at the comments and messages that we received from members of the public who connected with our research online. 
Blog Post Editing Process

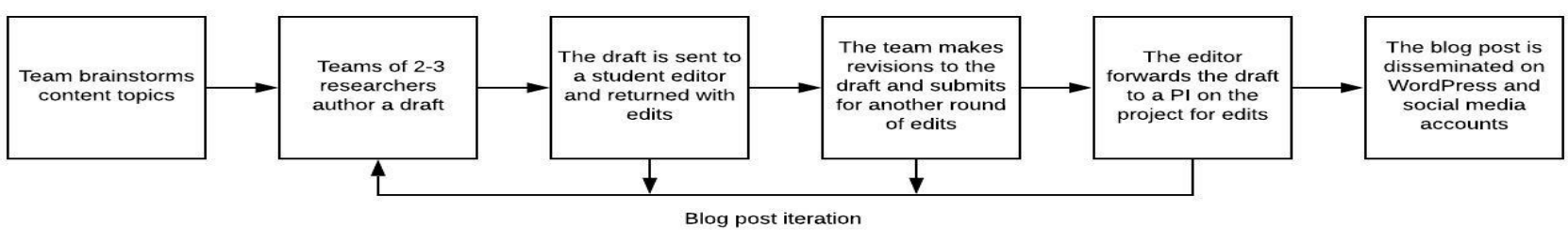

Figure 2: A diagram of the blog post editing process, beginning with content creation and ending with dissemination on social media.

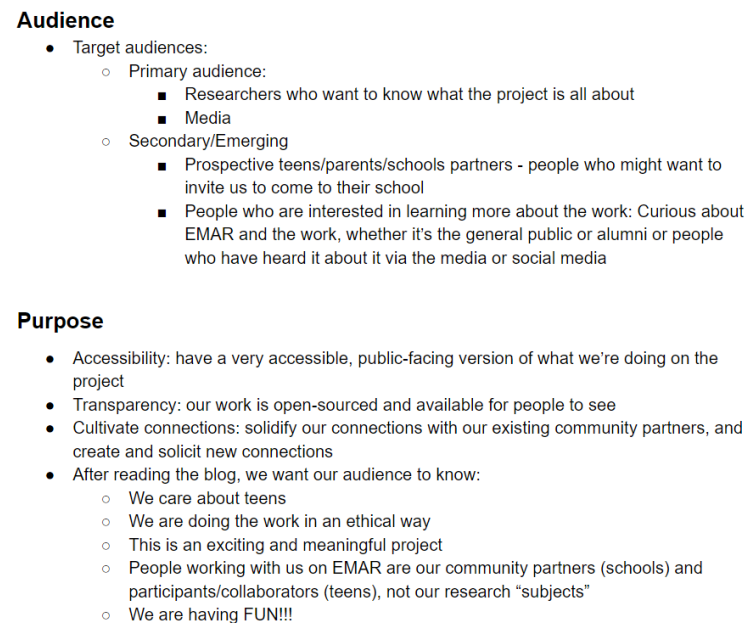

Figure 3: The blog post style guide is a living document created in Google Documents, giving authors insight into audience and tone of voice.

\subsection{Audience experiences}

Our team tracked WordPress views over the course of the 2018 academic year to gain an understanding of how users were interacting with our blog post content. Our team posted three blogs during February and five blogs during March to our WordPress account, sharing our blog posts on our Facebook and Twitter pages. This was a period of time in which our research team was hosting a large community event at our university, and we were working with seven local area high schools in preparation for the event. This period of time in which we produced our highest number of blogs per month generated our highest number of website views. During February, we accumulated 418 views while in March we accumulated 701 views. This information suggests that blog traffic increased as the result of our increased number of blogs posted during this time frame.

Following this high traffic period of time on our blog site, our team did not post any blogs between May and June. As a result, our blog traffic decreased in May to 154 views. Views on our WordPress

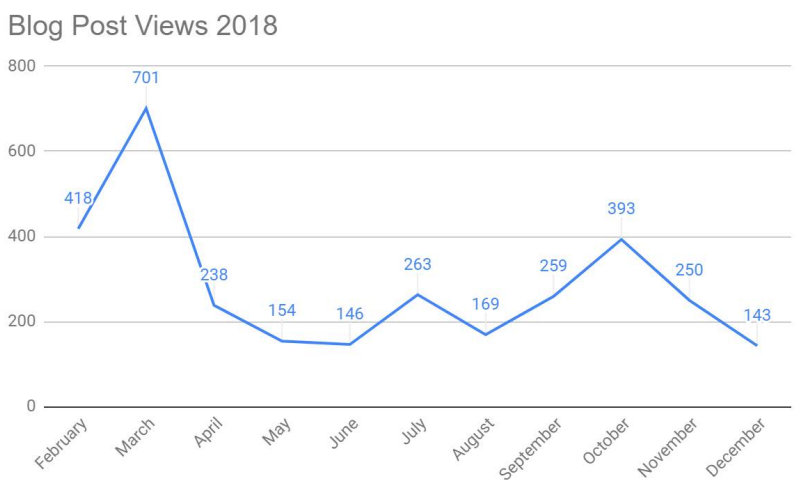

Figure 4: Blog post views by month during the 2018 year.

account continued to remain low through September as our team only posted two blogs during the five month stretch.

In sharing our blog post updates on our social media pages including Twitter and Facebook, our team was able to observe user responses by tracking comments, retweets and shares as well as likes and favorites. From July 2018 to April 2019, our blog posts received an average of six likes and two retweets per post. Our community partners, including the local schools and teachers that we have worked with, are among the users that have interacted with our posts on social media. In addition, other social media accounts from our university have engaged with our work on this platform.

When sharing our blog posts to Twitter, the community partners we have worked with have commented on our blogs and thanked us for our partnership and encouraged us to return to the field to introduce additional outreach activities to local students.

Further, there have been several instances where community members from new organizations have reached out to us on social media.

\subsection{Amplifying engagement}

Sharing our research on social media platforms accessible by the general public has allowed our team to connect with internal media sources at our university as well as external media sources who have been interested in learning more about our project. 
In regularly updating the public on our research efforts in the community, we have been contacted by members of the media team at our university to participate in interviews for publication on our university website. Student researchers have also been interviewed independently because of their involvement on the research project. By posting updates on our involvement in the community and integrating relevant hashtags in our social media posts, external media sources have been able to find and read our blog posts as well as connect with our research findings to understand the progress that we have made in developing a social robot that will address teen stress. As a result of sharing our blog posts on social media, our team has been invited to share our story with news sources, as well as present our findings and long-term research goals on podcasts.

\subsection{On-boarding}

In addition to sharing information and details about the research with our community partners, maintaining a research blog allows our team to use the blog as a resource to on-board new team members interested in working on the project. The blog provides new researchers with comprehensive access to our previous research activities and findings and providing background on how the research has developed overtime. It helps to further instantiate the values of our project which stress interdisciplinarity, transparency, and reciprocity. Students who wish to join or who have recently joined Project EMAR can read the archives to get sense of what previous work we have done and also how we do that work.

\section{REFLECTIONS}

In this section, we discuss the successes and challenges we have encountered in using blogging as a research dissemination tool.

\subsection{Successes}

Using blogging to disseminate our research findings has allowed our team to represent each of the unique perspectives of our team members while engaging our community partners in the design process. Having each of our researchers participate in the process of authoring a blog has allowed our team to ensure that each step in the design process is reflected in the findings we share with the public. It helps create ownership of the research we are doing and the relationships we are building with partners.

In addition, sharing our findings on social media has allowed us to connect with members in the community who would be unable to access our research findings in an academic journal. In disseminating our blogs on social platforms including Twitter and Facebook, our team is able to engage with community members using like and dislike functions, as well as comments sections. In doing so, we are able to maintain a participatory and ethical relationship with our community partners as we provide real-time updates on our research progress.

Creating a research blog has also benefited members of our team because student researchers participate in the process of writing and publishing blogs based on their experiences in the field. Writing content that aims to engage community members in our research process allows our student researchers to reflect on the design and data collection process. Blogging allows students to empathize with the needs of community members to see the connection between our research and the relationship it has to the communities we work with.

In regularly producing blog posts throughout the year, student authors are given a byline for their professional portfolios that they can use to talk about the work they have completed during their academic career. Students have used their blog posts as examples of the research and writing skills they have obtained during their time at the university in interviews for professional jobs and internships. Students have voiced that they appreciate the process of authoring blog posts because it gives them a deeper understanding of the work the research they have performed in the community and how they can articulate their research process to a general audience. Further, other team members have mentioned they appreciated the opportunity for engaging in public writing and having the chance to work with editors and revision which has in turn helped to strengthen their writing.

\subsection{Challenges}

While blogging is effective in connecting our community partners to our latest research findings, it can be challenging to maintain a consistent blogging schedule. One challenge is that students are usually heads down on the research work in the quarter which results in a larger number of completed blog posts at the end of the term. Further, it is challenging to post up-to-date content during campus-wide breaks in the academic year. During breaks in the academic year, our blog and social media pages are not updated frequently and can go for extended periods of time without sharing new content with the community. To address these issues, we are trying to be more intentional with the timing and save up some of the blog posts that accumulate at the end of a term and space them out over time.

Blogging is a time-consuming process that involves authors spending time communicating about the research that is being done while it is taking place. As a result, publishing regular content during busy times in the research year can make it difficult to provide constant updates on the team's progress and findings. Our team incorporates the voices of all researchers on our team to make the writing and editing process manageable, such that we can regularly produce content throughout the year. In creating a blog post schedule and asking team members to sign up to author a blog in groups of 2-3 students, the labor involved in blogging is distributed as multiple team members share the responsibility of completing the work. This can be especially helpful for team members who might be reticent to post their writing online, because working as a team can reduce the anxiety of writing and publishing for emerging writers and also strengthen the final product.

In addition, maintaining consistency across the blog posts can be difficult because blog posts are authored by a diverse group of researchers throughout the academic year. As a result, it is difficult to maintain a consistent tone across our social channels and blog posts because authors communicate and share research results in different ways. To address this issue, our team uses a blog post style guide to answer author's questions involving target audience, tone, style, formatting, and media integration. In addition, having a team of editors that revise and edit each blog post for consistency 
in voice and tone allows each post to remain representative of the research team's authentic tone of voice.

\subsection{Best practices and takeaways}

Blogging is a beneficial tool for researchers to use to disseminate research findings to interested members of the public. To maintain the effectiveness of blogging, it is important that teams ensure that blog posts go through the editing process to maintain consistency in tone and style. Having a team of editors responsible for reading each blog post prior to sharing them online ensures that posts will capture the voice of the research team.

6.3.1 Multiple authors. Authoring blog posts in teams allows researchers to capture multiple perspectives of the research process, providing a holistic view of fieldwork. On our interdisciplinary team of researchers, each student brings a unique perspective to design and research. Having students go through the process of writing and editing collaboratively allows students to share their point of view with their team members while providing our audience with a view into the different areas of our research.

6.3.2 Making posts visually engaging. Blog posts that are visually engaging allow audiences to connect to the research that is being done within the community. By integrating visuals from our fieldwork with our community partners, our audience is able to see research in action. These visuals also allow the parents of the teens we work with to see what activities their children are participating in.

6.3.3 Amplifying on social media. Disseminating blogs on social media platforms has allowed our team to connect with community members who would be unlikely to access our research in an academic journal. Sharing our blog posts on Twitter and Facebook has allowed our team to gain feedback from our audience in real-time on our posts through the ability for users to like, share, and comment on posts. This communicative function of social media allows our team to share our research with members of the public in an accessible way while providing our community partners with ways to connect with our research.

6.3.4 Scheduling for slow times. Because the blog posts are authored by student researchers, we encountered lulls in the academic year that prevented us from producing blog post content during campus-wide breaks. In anticipation of these slow points in the year, our team created a collaborative blog post schedule to ensure that content would be prepared in advance for periods in which research would be lacking. In preparing content for times in which research would be limited, we were able to continue to engage our community partners in the research process.

6.3.5 Accessibility. It is also essential that members of the editing team are critically aware of how authors can write web content that is accessible to all audiences. Editors need to follow best practices in accessibility including using semantic structure by using headings that convey both meaning and structure and can organize the content. Also, when integrating images into blog posts, all images need to have alt text to aid users who would be using a screen reader when accessing the blog. Integrating formatting for accessibility into the blog post style guide will allow authors to produce blog content that meets the needs of all audience members. Blog posts should be checked to ensure they meet accessibility requirements.

6.3.6 Style guide. Using a blog post style guide has allowed our team to maintain consistency across the blogging platform and produce posts that are consistent in terms of style and voice. However, overtime, the style guide needs to be modified to best meet the growing needs of users. Taking this into consideration, the blog post style guide that our team created is a living document that is constantly iterated on as questions come up on the research team. Maintaining the blog post style guide in a Google Document allows our team to make frequent updates that are shared with the larger team to communicate best practices as necessary.

\section{CONCLUSION}

In conclusion, blogging has the potential to play a fundamental role in engaging communities in the research process. By using blogging as a research dissemination tool, researchers can quickly connect their audience with real-time insights and reflections at all steps in the research process to create a transparent and reciprocal research relationship.

\section{ACKNOWLEDGMENTS}

We wish to thank our community partners and schools who engaged in our research project. Thanks to Elin Björling, Maya Cakmak, and the entire team of student researchers on Project EMAR who have contributed their ideas, words, and images to the blog over the past three years. This research was funded in part by the National Science Foundation's National Robotics Initiative grant No. NRI:INT 1734100 .

\section{REFERENCES}

[1] American Psychological Association (2014). Stress in America 2013 highlights: Are teens adopting adults stress habits.

[2] Amanda Cooper. 2014. The use of online strategies and social media for research dissemination in education. Education Policy Analysis Archives 22 (2014), 88.

[3] Noelle R. Leonard, Marya V. Gwadz, Amanda Ritchie, Jessica L. Linick, Charles M. Cleland, Luther Elliott, and Michele Grethel. 2015. A multi-method exploratory study of stress, coping, and substance use among high school youth in private schools. Frontiers in psychology 6 (2015), 1028.

[4] Kostas Metaxiotis, Kostas Ergazakis, and John Psarras. 2005. Exploring the world of knowledge management: Agreements and disagreements in the academic/practitioner community. Fournal of knowledge management 9, 2 (2005), 6-18.

[5] Douglas A Powell, Casey J Jacob, and Benjamin J Chapman. 2012. Using blogs and new media in academic practice: Potential roles in research, teaching, learning, and extension. Innovative Higher Education 37, 4 (2012), 271-282.

[6] Emma J Rose and Elin A Björling. 2017. Designing for engagement: Using participatory design to develop a social robot to measure teen stress. In Proceedings of the 35th ACM International Conference on the Design of Communication. ACM, 7.

[7] Katy Schnitzler, Nigel Davies, Fiona Ross, and Ruth Harris. 2016. Using Twitter to drive research impact: A discussion of strategies, opportunities and challenges. International Journal of Nursing Studies 59 (2016), 15-26.

[8] Saul Shiffman, Arthur A Stone, and Michael R Hufford. 2008. Ecological momentary assessment. Annu. Rev. Clin. Psychol. 4 (2008), 1-32. 